\title{
PLASMA EMISSION BY COUNTER-STREAMING ELECTRON BEAMS
}

\author{
L. F. Ziebell ${ }^{1}$, L. T. Petruzzellis ${ }^{1}$, P. H. Yoon ${ }^{2,3}$, R. Gaelzer ${ }^{1}$, and J. Pavan ${ }^{4}$ \\ ${ }^{1}$ Instituto de Física, UFRGS, Porto Alegre, RS, Brazil; luiz.ziebell@ufrgs.br \\ 2 Institute for Physical Science \& Technology, University of Maryland, College Park, MD, USA; yoonp@umd.edu \\ ${ }^{3}$ School of Space Research, Kyung Hee University, Yongin, Korea \\ ${ }^{4}$ Instituto de Física e Matemática, UFPel, Pelotas, RS, Brazil; joel.pavan@ufpel.edu.br \\ Received 2015 November 6; accepted 2015 December 17; published 2016 February 8
}

\begin{abstract}
The radiation emission mechanism responsible for both type-II and type-III solar radio bursts is commonly accepted as plasma emission. Recently Ganse et al. suggested that type-II radio bursts may be enhanced when the electron foreshock geometry of a coronal mass ejection contains a double hump structure. They reasoned that the counter-streaming electron beams that exist between the double shocks may enhance the nonlinear coalescence interaction, thereby giving rise to more efficient generation of radiation. Ganse et al. employed a particle-in-cell simulation to study such a scenario. The present paper revisits the same problem with EM weak turbulence theory, and show that the fundamental $(F)$ emission is not greatly affected by the presence of counter-streaming beams, but the harmonic $(H)$ emission becomes somewhat more effective when the two beams are present. The present finding is thus complementary to the work by Ganse et al.
\end{abstract}

Key words: plasmas - radiation mechanisms: non-thermal - solar wind - Sun: radio radiation - turbulence - waves

\section{INTRODUCTION}

Solar radio bursts (Wild 1950a, 1950b; Wild \& McCready 1950; Wild et al. 1954) have been studied observationally and theoretically for over six decades. Of the five different types of solar radio bursts that have been identified to date, type-III radio bursts are perhaps the best studied and understood. For historical and recent reviews, see, e.g., Ginzburg \& Zheleznyakov (1958), Wild et al. (1963), Kundu (1965), Tsytovich (1967), Zheleznyakov (1970), Wild \& Smerd (1972), Stewart (1974), Fainberg \& Stone (1974), Lin (1974), Smerd (1976), Rosenberg (1976), Melrose (1980a, 1980b, 1986), Goldman (1983), Suzuki \& Dulk (1985), Dulk (1985), Reiner et al. (1992, 2009), Robinson \& Cairns (1998a, 1998b, 1998c), Cane et al. (2002), Reid \& Ratcliffe (2014), Ziebell et al. (2014, 2015).

The general consensus is that the radiation emission mechanism responsible for type-III bursts is plasma emission, first proposed by Ginzburg \& Zheleznyakov (1958) and subsequently refined over the intervening decades (Tsytovich 1967; Kaplan \& Tsytovich 1968; Zheleznyakov \& Zaitsev 1970a; Melrose 1982, 1987, Cairns 1987a, 1987b, 1987c; Goldman \& Dubois 1982; Robinson \& Cairns 1998a, 1998b, 1998c; Li et al. 2005a, 2005b, 2006a, 2006b, 2008a, 2008b, 2009, 2010, 2011a, 2011b, 2011c; Ratcliffe et al. 2012; Reid \& Ratcliffe 2014; Ziebell et al. 2014, 2015). In addition to theory, full particle simulations of the plasma emission process have also been carried out (Kasaba et al. 2001; Karlický \& Vandas 2007; Rhee et al. 2009a, 2009b; Umeda 2010; Ganse et al. 2012a, 2012b). The emission of radiation at the local plasma frequency and/or its harmonic(s), hence "plasma" emission, is a result of nonlinear partial conversion of kinetic energy of electron beams into EM radiation energy, but the process involves sophisticated intermediate steps. The first step involves the excitation of Langmuir $(L)$ waves via the wellknown bump-on-tail instability when the energetic electron stream produced at the site of the solar flare interacts with the background solar wind plasma as the stream flows outward along open magnetic field lines. The next step involves nonlinear processes of wave decay and scattering, which eventually lead to the generation of radiation.

Type-II radio bursts are related to type-III bursts in that the underlying radiation emission mechanism is the same for both types of burst, namely plasma emission (Roberts 1959; Wild et al. 1959; Cane et al. 1987). The commonly accepted scenario for type-II bursts involves the acceleration of electrons at the shock front generated by a coronal mass ejection (CME). The electrons are accelerated in the CME foreshock region and form a (generally non-gyrotropic) beam distribution, which then undergoes the customary beam-plasma interaction (Karlický \& Vandas 2007). The ensuing nonlinear processes are expected to be similar to those in the type-III situation.

The distinguishing characteristic associated with type-II radio bursts is the fundamental/harmonic $(F / H)$ two-band structure. While some type-III bursts also feature such $F / H$ structure, they are more commonly observed in a single band, or at least it is difficult to distinguish the two-band feature owing to the fast frequency drift rate. The two-band frequency structure is generally interpreted in terms of an electron beam accelerated at the front of the CME shock, which then excites Langmuir waves by the beam-plasma instability, followed by partial nonlinear conversion to the radiation modes at the fundamental and (second) harmonic plasma frequencies. The recent calculation of EM weak turbulence by Ziebell et al. $(2014,2015)$ is consistent with such an interpretation, where it is shown that the emissions at the fundamental $(F)$ and harmonic $(H)$ commonly take place together in association with the single beam-plasma system, but rarely just a single band, either $F$ or $H$. Ziebell et al. $(2014,2015)$ also demonstrate that radiation emission at the third harmonic or higher is also extremely rare.

The present paper is concerned with an important point raised by Ganse et al. (2012a, 2012b), who noted that the CME shock front spans a large spatial area, which encompasses greatly varying local plasma frequency. If the beam-generated plasma emission should occur at every point associated with the CME shock front, then the type-II bursts should be observed in a broad frequency band. Nevertheless, type-II radio emissions are invariably narrow-band. Consequently, these 
authors theorize that the actual type-II bursts might be related to special circumstances in the CME foreshock region where the shock front geometry is such that it forms a double hump. They reason that the radio emission might preferentially take place within the depression region sandwiched between the two shock fronts where there are counter-streaming electron beams. In order to demonstrate their scenario quantitatively, Ganse et al. (2012a, 2012b) carried out a particle-in-cell (PIC) simulation and showed that the plasma emission is enhanced for the case of counter-streaming beams when compared with a single beam. A similar result regarding the enhancement of $H$ emission in the case of counter-streaming electron beams was also obtained by Umeda (2010).

The present paper revisits the problem of plasma emission by counter-streaming electron beams, which Ganse et al. (2012a, 2012b) and Umeda (2010) investigated by means of PIC code simulation, but here we employ the numerical solution of EM weak turbulence theory. The present investigation complements the previous studies by Ganse et al. (2012a, 2012b) and Umeda (2010). As we shall discuss, our findings are largely consistent with the above-mentioned earlier works, but we also provide greater details. The present weak turbulence simulation naturally lends itself to detailed theoretical analysis, since unlike the brute-force method of PIC code simulation, EM weak turbulence simulation is based upon theoretical equations, which contain various terms that can be identified with specific physical processes. Consequently, by artificially turning certain terms on or off, one may unequivocally identify what specific terms in the equation are responsible for what physical process, as Ziebell et al. (2015) have done. The EM weak turbulence method is also computationally far more efficient than direct PIC code simulation, and thus one may survey a larger input parameter space, which is what we have done in the present paper.

The organization of the present paper is as follows: in Section 2 we briefly describe the theoretical formulation and numerical setup. Section 3 presents and discusses the results of numerical solutions. Finally, Section 4 concludes the paper and comments on the results obtained.

\section{THEORETICAL FORMULATION AND NUMERICAL SETUP}

The basic theoretical framework and the foundational set of equations for EM weak turbulence theory were introduced in our recent papers (Ziebell et al. 2014, 2015). We briefly note that we are concerned with three basic plasma eigenmodes: Langmuir $(L)$, ion-sound $(S)$, and transverse $(T)$ modes. The energy density of the longitudinal electric field thus consists of contributions from $L$ and $S$ modes, $\left\langle\delta E_{\|}^{2}\right\rangle_{k, \omega}=\sum_{\sigma= \pm 1} \sum_{\alpha=L, S} I_{k}^{\sigma \alpha} \delta\left(\omega-\sigma \omega_{k}^{\alpha}\right)$, while the spectral energy density of the transverse electric field is defined in terms of the $T$ mode, $\left\langle\delta E_{\perp}^{2}\right\rangle_{k \omega}=\sum_{\sigma= \pm 1} I_{k}^{\sigma T} \delta\left(\omega-\sigma \omega_{k}^{T}\right)$. The magnetic field intensity is related to the spectral energy density of the transverse $E$ field by $\left\langle\delta B^{2}\right\rangle_{k \omega}=|c k / \omega|^{2}\left\langle\delta E_{\perp}^{2}\right\rangle_{k \omega}$. Linear dispersion relations for $L, S$, and $T$ modes are given by textbook results, $\quad \omega_{k}^{L}=\omega_{p e}\left(1+3 k^{2} \lambda_{D}^{2}\right)^{1 / 2}, \quad \omega_{k}^{S}=k c_{S}\left(1+3 T_{i} / T_{e}\right)^{1 / 2}$ $\left(1+k^{2} \lambda_{D}^{2}\right)^{-1 / 2}$, and $\omega_{k}^{T}=\left(\omega_{p e}^{2}+c^{2} k^{2}\right)^{1 / 2}$, respectively, where $\omega_{p e}=\left(4 \pi n_{e} e^{2} / m_{e}\right)^{1 / 2}$ is the plasma frequency, $n_{e}, e$, and $m_{e}$ being the electron number density, unit electric charge, and electron mass, respectively. The Debye length is defined by
$\lambda_{D}=\left(T_{e} / 4 \pi n_{e} e^{2}\right)^{1 / 2}=v_{t h} /\left(\sqrt{2} \omega_{p e}\right), T_{e}$ and $T_{i}$ being electron and ion temperatures, respectively. Thermal speed $v_{t h}=\left(2 T_{e} / m_{e}\right)^{1 / 2}$ and ion-sound speed $c_{S}=\left(T_{e} / m_{i}\right)^{1 / 2}$ are also defined, $m_{i}$ being the ion (proton) mass.

The basic wave kinetic equations governing the time evolution of $L, S$, and $T$ modes, as well as the particle kinetic equation for the electron distribution function, are given in Ziebell et al. (2014, 2015), so they will be presented here only in condensed form. We employ the normalized quantities and definitions introduced in Ziebell et al. (2014, 2015): $z=\omega / \omega_{p e}, \tau=\omega_{p e} t$, $\boldsymbol{q}=\boldsymbol{k} v_{t h} / \omega_{p e}, \quad \boldsymbol{u}=\boldsymbol{v} / v_{t h}, \quad \mu_{q}^{L}=1, \quad \mu_{q}^{T}=1, \quad \mu_{q}^{S}=q^{3} / 2^{3 / 2}$ $/\left(\sqrt{m_{e} / m_{i}} / \sqrt{1+3 T_{i} / T_{e}}\right), g=\left[2^{3 / 2}(4 \pi)^{2} n_{e} \lambda_{D e}^{3}\right]^{-1}$, where $z$, $\tau, \boldsymbol{q}$, and $\boldsymbol{u}$ represent normalized frequency, time, wavevector, and velocity vector, respectively. The other quantities, $\mu_{q}^{L}, \mu_{q}^{T}$, and $\mu_{q}^{S}$, are auxiliary dimensionless quantities, and $g$ is inversely proportional to the number of particles in a sphere with radius equal to the Debye length. This quantity must be sufficiently small in order for the ionized gas to qualify as a plasma. The normalized particle velocity distribution function and normalized wave spectra are defined by

$$
\Phi_{a}(\boldsymbol{u})=v_{t h}^{3} F_{a}(\boldsymbol{v}), \quad \mathcal{E}_{q}^{\sigma \alpha}=\frac{(2 \pi)^{2} g}{m_{e} v_{t h}^{2}} \frac{I_{k}^{\sigma \alpha}}{\mu_{k}^{\alpha}} .
$$

The equations of EM weak turbulence theory in nondimensional forms are given as follows. First, the wave kinetic equations for $L, S$, and $T$ modes, in condensed form, are

$$
\begin{aligned}
\frac{\partial \mathcal{E}_{q}^{\sigma L}}{\partial \tau}= & \left.\frac{\partial \mathcal{E}_{q}^{\sigma L}}{\partial \tau}\right|_{L q l}+\left.\frac{\partial \mathcal{E}_{q}^{\sigma L}}{\partial \tau}\right|_{L d L S}+\left.\frac{\partial \mathcal{E}_{q}^{\sigma L}}{\partial \tau}\right|_{L s L L}, \\
\frac{\partial \mathcal{E}_{q}^{\sigma S}}{\partial \tau}= & \left.\frac{\partial \mathcal{E}_{q}^{\sigma S}}{\partial \tau}\right|_{S q l}+\left.\frac{\partial \mathcal{E}_{q}^{\sigma S}}{\partial \tau}\right|_{S d L L}, \\
\frac{\partial \mathcal{E}_{q}^{\sigma T}}{\partial \tau}= & \left.\frac{\partial \mathcal{E}_{q}^{\sigma T}}{\partial \tau}\right|_{T d L L}+\left.\frac{\partial \mathcal{E}_{q}^{\sigma T}}{\partial \tau}\right|_{T d L S} \\
& +\left.\frac{\partial \mathcal{E}_{q}^{\sigma T}}{\partial \tau}\right|_{T d T L}+\left.\frac{\partial \mathcal{E}_{q}^{\sigma T}}{\partial \tau}\right|_{T s T L} .
\end{aligned}
$$

Detailed expressions for the different terms appearing in these equations can be seen, for instance, in Ziebell et al. (2014, 2015).

The particle kinetic equation in normalized form is as follows:

$$
\begin{aligned}
\frac{\partial \Phi_{a}(\boldsymbol{u})}{\partial \tau}= & \frac{e_{a}^{2}}{e^{2}} \frac{m_{e}^{2}}{m_{a}^{2}} \\
& \times \sum_{\sigma} \sum_{\alpha=L, S} \int d \boldsymbol{q}\left(\frac{\boldsymbol{q}}{q} \cdot \frac{\partial}{\partial \boldsymbol{u}}\right) \mu_{q}^{\alpha} \delta\left(\sigma z_{q}^{\alpha}-\boldsymbol{q} \cdot \boldsymbol{u}\right) \\
& \times\left(g \frac{m_{a}}{m_{e}} \frac{\sigma z_{q}^{L}}{q} \Phi_{a}(\boldsymbol{u})+\mathcal{E}_{q}^{\sigma \alpha} \frac{\boldsymbol{q}}{q} \cdot \frac{\partial \Phi_{a}(\boldsymbol{u})}{\partial \boldsymbol{u}}\right) .
\end{aligned}
$$

In the above $a=e$ denotes the electrons, and $a=i$ stands for the ions (protons). The dispersion relations for plasma normal modes 
that appear in these equations are now given in dimensionless form by $z_{q}^{L}=\left(1+3 q^{2} / 2\right)^{1 / 2}, z_{q}^{S}=q A /\left(1+q^{2} / 2\right)^{1 / 2}, z_{q}^{T}=$ $\left(1+c^{2} q^{2} / v_{t e}^{2}\right)^{1 / 2}$, where

$$
A=\frac{1}{\sqrt{2}}\left(\frac{m_{e}}{m_{i}}\right)^{1 / 2}\left(1+\frac{3 T_{i}}{T_{e}}\right)^{1 / 2}
$$

In Equation (1) for the $L$ mode, the first term, designated as $L q l$, describes the spontaneous and induced emissions, which are quasilinear effects. The second term, denoted by $L d L S$, describes three-wave decay processes involving $L$ and $S$ waves. The third term, denoted as $L s L L$, represents the effects of nonlinear wave-particle scattering involving two $L$ waves and particles. The equation for an $S$ wave in (1) contains the first term denoted by $S q l$, which describes quasilinear processes of spontaneous and induced emissions. The second term, designated by $S d L L$, describes the three-wave decay involving an $S$ mode and two $L$ waves. For the $T$ mode, the first term describes the merging of two $L$ waves into a $T$ mode at approximately twice the plasma frequency (the harmonic or $H$ emission). This wave-wave merging term is the opposite of a decay process, and is thus denoted as $T d L L$. The second term, TdLS, describes the influence of the decay process of an $L$ mode into a $T$ mode at approximately the fundamental plasma frequency and an $S$ wave, which is one of the processes responsible for the fundamental $(F)$ emission. The third term, denoted by $T d T L$, characterizes three-wave merging (the opposite of decay) of one $L$ mode wave and a $T$ mode into a higher-harmonic $T$ mode. The last term, TsTL, describes nonlinear scattering involving an $L$ mode into a $T$ mode at the fundamental mediated by the particles. This is another process responsible for the fundamental plasma emission. The particle kinetic Equation (2) is given in the form of a Fokker-Planck equation, which contains the velocity friction and diffusion terms on the right-hand side. In both wave and particle equations, terms associated with the parameter $g$ describe effects that arise owing to spontaneous thermal fluctuations.

The numerical routine to solve the complete set of dimensionless Equations (1) and (2) in two-dimensional (2D) wavenumber space and 2D velocity space employs a splitting method with fixed time step for the evolution of the distribution and a Runge-Kutta method with the same fixed time step for the wave equations. The initial configurations are such that ions are considered stationary, and electrons as well as the waves evolve in time. The ion distribution in $2 \mathrm{D}$ velocity space in dimensionless form is given by

$$
\Phi_{i}(\boldsymbol{u})=\frac{1}{\pi} \frac{T_{e}}{T_{i}} \frac{m_{i}}{m_{e}} \exp \left(-\frac{m_{i}}{m_{e}} \frac{T_{e}}{T_{i}} u^{2}\right) .
$$

The initial electron distribution function is assumed to be made of a Maxwellian background population and a pair of forward and backward beam components, with number densities assigned by $n_{f}$ and $n_{b}$. In 2D it is given in dimensionless variables as follows:

$$
\begin{aligned}
\Phi_{e}(\boldsymbol{u}, 0)= & \frac{1}{\pi}\left(1-\frac{n_{f}}{n_{0}}-\frac{n_{b}}{n_{0}}\right) \exp \left[-u_{\perp}^{2}-\left(u_{\|}-\frac{v_{0}}{v_{t e}}\right)^{2}\right] \\
& +\frac{1}{\pi} \frac{n_{f}}{n_{0}} \frac{T_{e}}{T_{f}} \exp \left\{-\frac{T_{e}}{T_{f}}\left[u_{\perp}^{2}+\left(u_{\|}-\frac{v_{f}}{v_{t f}}\right)^{2}\right]\right\} \\
& +\frac{1}{\pi} \frac{n_{b}}{n_{0}} \frac{T_{e}}{T_{b}} \exp \left\{-\frac{T_{e}}{T_{b}}\left[u_{\perp}^{2}+\left(u_{\|}-\frac{v_{f}}{v_{t b}}\right)^{2}\right]\right\} .
\end{aligned}
$$

Here, $\quad v_{t e}=\left(2 T_{e} / m_{e}\right)^{1 / 2}, \quad v_{t f}=\left(2 T_{f} / m_{e}\right)^{1 / 2}, \quad$ and $\quad v_{t b}=$ $\left(2 T_{b} / m_{e}\right)^{1 / 2}$ are background, forward-beam, and backwardbeam thermal speeds, respectively, and $v_{0}, v_{f}$, and $v_{b}$ are drift velocities associated with the background, forward, and backward beams, respectively. The drift velocity for the background $v_{0}$ is chosen such that it guarantees zero net drift velocity for the total electron distribution, i.e., $v_{0}=$ $-\left(n_{f} v_{f}+n_{b} v_{b}\right) /\left(n_{0}-n_{f}-n_{b}\right)$.

The intensities of $L$ and $S$ waves are initialized by balancing the spontaneous and induced emissions, taking into account the background population. The normalized spectral intensities of the electric field are therefore given by

$$
\begin{aligned}
& \mathcal{E}_{q}^{\sigma L}(0)=\frac{g}{2\left(z_{q}^{L}\right)^{2}}, \\
& \mathcal{E}_{q}^{\sigma S}(0)=\frac{g}{2 z_{q}^{L} z_{q}^{S}} \frac{\exp \left(-\xi_{q}^{2}\right)+\eta^{1 / 2} \exp \left(-\eta \xi_{q}^{2}\right)}{\exp \left(-\xi_{q}^{2}\right)+\left(T_{e} / T_{i}\right) \eta^{1 / 2} \exp \left(-\eta \xi_{q}^{2}\right)}, \\
& \xi_{q}^{2}=\frac{\left(z_{q}^{S}\right)^{2}}{q^{2}}, \quad \eta=\frac{m_{i}}{m_{e}} \frac{T_{e}}{T_{i}} .
\end{aligned}
$$

The $T$ waves are assumed to be non-existent at the initial time. Note that the forward-propagating $(\sigma=+1)$ and backwardpropagating $(\sigma=-1)$ initial spectra are assumed to be the same, that is, they are initially isotropic in $q$.

In the numerical analysis we adopt the normalized time interval $\Delta \tau=0.1$. We employ $51 \times 51$ grids for $q_{\perp}$ and $q_{\|}$, with $0<q_{\perp}=k_{\perp} v_{t e} / \omega_{p}<0.6$ and $0<q_{\|}=k_{\|} v_{t e} / \omega_{p}<0.6$. For the velocities, we use $63 \times 125$ grids for $\left(u_{\perp}, u_{\|}\right)=\left(v_{\perp} / v_{t h}, v_{\|} / v_{t h}\right)$, covering the velocity range $0<u_{\perp}=v_{\perp} / v_{t h}<15$ and $-15<u_{\|}=v_{\|} / v_{t h}<15$. For the subsequent numerical solutions we assume that forward and backward beams have the same thermal spread as the background so that $T_{f} / T_{e}=1=T_{b} / T_{e}$. The electron-to-ion temperature ratio of $T_{e} / T_{i}=7$ is used, and the plasma parameter $\left(n_{0} \lambda_{D}^{3}\right)^{-1}=5 \times 10^{-3}$ is adopted. We also assume $v_{t e}^{2} / c^{2}=4.0 \times 10^{-3}$.

The sizes of the grids used for the numerical analyses are chosen as a compromise between the resolution obtained and the performance of the numerical code. In wavenumber space the grid allows for a resolution that is sufficient to show fundamental features of the wave spectra, such as the primary and backward peaks of $L$ waves and the peaks in the $T$ wave spectra that are identified as the fundamental and harmonic emissions. In velocity space, the grid used allows for a smooth description of the background distribution function and of the beam. Using these grid sizes, the code needs to run for a few 

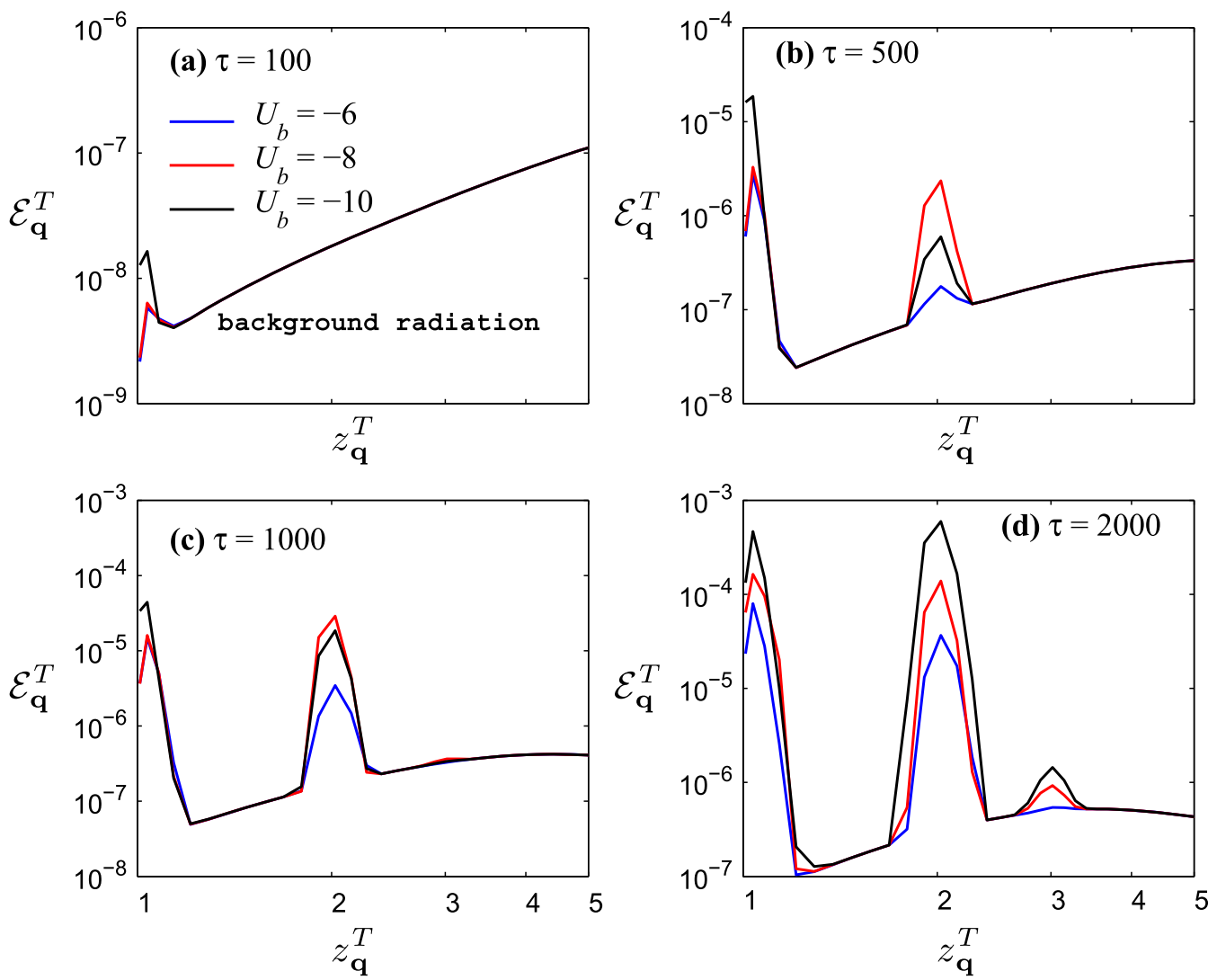

Figure 1. Normalized wave intensity for $T$ waves, $\mathcal{E}_{q}^{T}$, as a function of normalized $T$ wave frequency, $z_{q}^{T}$. Ratios of beam densities are $N_{f}=6 \times 10^{-4}$ and $N_{b}=4 \times 10^{-4}$, forward beam speed is $U_{f}=8$, and three cases of backward beam speeds are used: $U_{b}=-6$ (blue), $U_{b}=-8$ (red), and $U_{b}=-10$ (black). The four panels are for different normalized time steps: (a) $\tau=100$; (b) $\tau=500$; (c) $\tau=1000$; and (d) $\tau=2000$.

days in a personal computer in order to obtain the results presented here. For 2D applications, a twofold increase in resolution would require nearly a fourfold increase in computational time. We have reached the conclusion that such costly enhanced resolution is not necessary to describe the relevant features of the time evolution of the system under investigation.

\section{NUMERICAL ANALYSIS}

For our numerical analysis we consider the case of a Maxwellian distribution for the background and a pair of counter-streaming beams, with total beam density $N_{f}+N_{b}=\left(n f+n_{b}\right) / n_{0}=1 \times 10^{-3}$, and normalized velocity of the forward beam $U_{f}=v_{f} / v_{t e}=8.0$.

The spectra of $L$ and $S$ waves are initialized according to Equation (5). The $T$ waves are initially absent. In Figures 1 and 2 we show the normalized wave intensity for the $T$ waves as a function of normalized wavenumber $q$, by integrating $\int d \theta q \mathcal{E}_{q}^{T}$, where $\theta=\cos ^{-1} q_{\|} / q$ and $q=\left(q_{\perp}^{2}+q_{\|}^{2}\right)^{1 / 2}$. In Figure 1, we show the results obtained in the case of a forward beam with a number density that is $50 \%$ higher than the number density of the backward beam, by assuming $N_{f}=6.0 \times 10^{-4}$ and $N_{b}=4.0 \times 10^{-4}$.

We investigate the effect of the variation of the velocity of the less dense beam, assuming $U_{f}=8.0$ and considering three values of backward beam velocity, namely, $U_{b}=$ $v_{b} / v_{t e}=-6.0,-8.0$, and -10.0 .
Figure 1(a) shows the normalized spectrum of $T$ waves, $\mathcal{E}_{q}^{T}$, obtained at $\tau=100$, for the three backward beam speeds. Even though the present runs assumed zero initial $T$ mode intensities, very early on a background radiation spectrum is generated, spanning the entire frequency range. This is the result of a newly discovered radiation emission mechanism that results from the nonlinear wave-particle scattering term TsTL, which is operative even for thermal electrons without beams (Ziebell et al. 2014, 2015). For the relative early time $\tau=100$, the growth of the peak corresponding to the fundamental emission of $T$ waves first appears. At this state, the fundamental emission is very weak, and the intensities are similar for the two cases of weaker beams, namely, $U_{b}=-6$ and $U_{b}=-8$, so that the two small peaks corresponding to these cases almost overlap. The case of $U_{b}=-10$, on the other hand, displays $F$ emission that is more enhanced compared to the cases of weaker backward beams. At this early time in the evolution, there is no indication of harmonic emission.

Figure 1(b) shows the result at $\tau=500$. The peaks corresponding to $F$ emission continue to grow with behavior similar to that observed in Figure 1(a), in the sense that the peaks corresponding to $U_{b}=-6$ and $U_{b}=-8$ are similar in height and spectral width, and that the peak for $U_{b}=-10$ is almost an order of magnitude higher than the other two. Note that $H$ emission is already quite significant at $\tau=500$. The harmonic emission is only incipient for the case of $U_{b}=-6$ (blue curve), but in the case of $U_{b}=-10$ (black), the harmonic mode attains an amplitude about 3-4 times higher than the case for $U_{b}=-6$. For $U_{b}=-8$ (red), on the other hand, the $H$ 

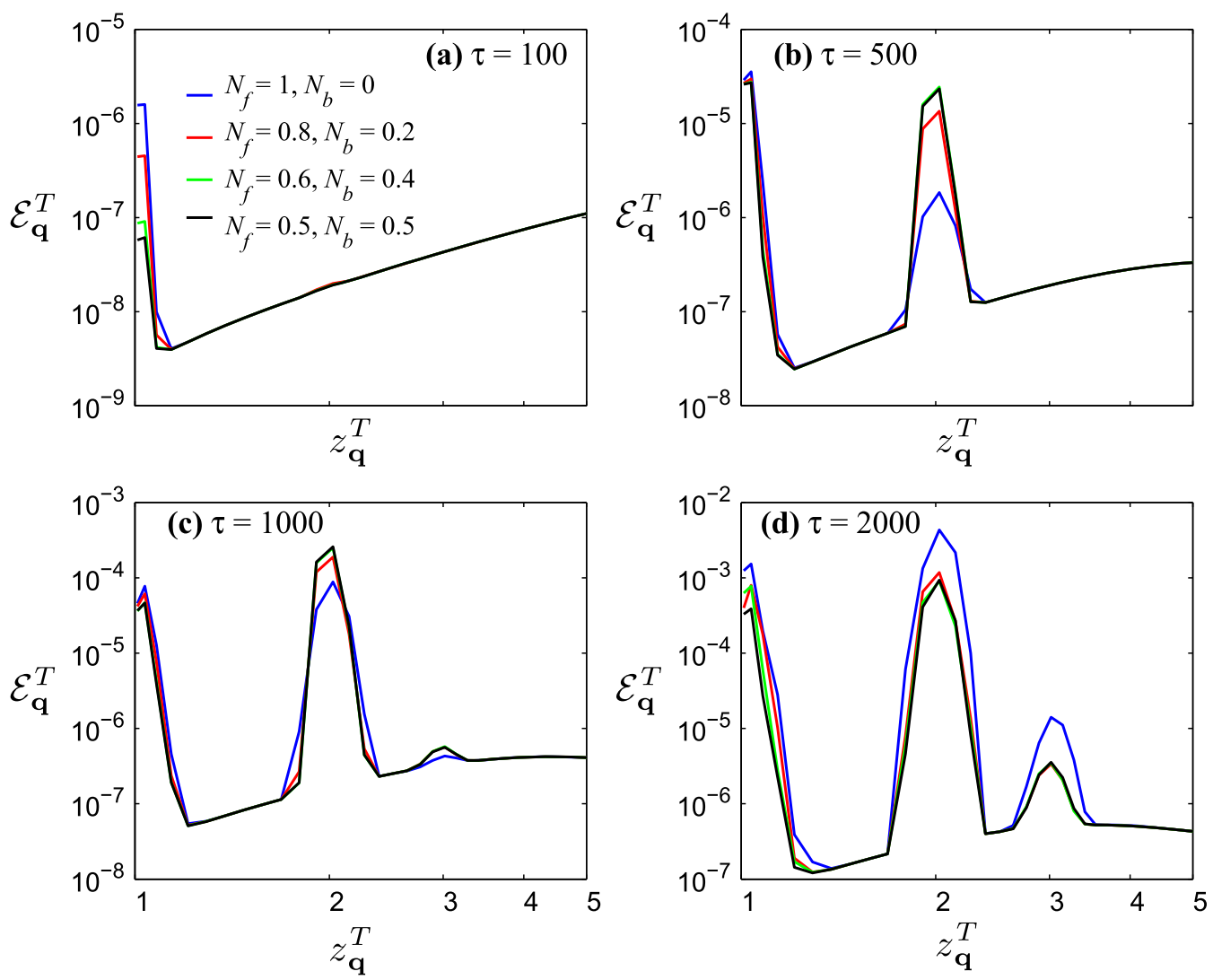

Figure 2. Normalized $T$ wave intensity, $\mathcal{E}_{q}^{T}$, as a function of normalized $T$ wave frequency, $z_{q}^{T}$. Forward and backward beam speeds are equal in magnitude, $U_{f}=10$ and $U_{b}=-10$, but four different combinations of the number density ratios are used: $N_{f}=1 \times 10^{-4}$ and $N_{b}=0$ (blue), $N_{f}=0.8 \times 10^{-4}$ and $N_{b}=0.2 \times 10^{-4}$ (red), $N_{f}=0.6 \times 10^{-4}$ and $N_{b}=0.4 \times 10^{-4}$ (green), and $N_{f}=0.5 \times 10^{-4}$ and $N_{b}=0.5 \times 10^{-4}$ (black). The four panels are for different normalized time steps: (a) $\tau=100 ;$ (b) $\tau=500 ;$ (c) $\tau=1000$; and (d) $\tau=2000$.

mode is almost an order of magnitude higher than for $U_{b}=-6$. This shows that $H$ emission grows more efficiently in the case of counter-propagating beams of equal speed rather than asymmetric speeds, while the efficiency of $F$ emission seems to be higher when the lower density beam has a greater speed than the higher density beam.

In the case of Figure 1(c), which corresponds to $\tau=1000$, the overall behavior is similar to that of Figure 1(b). The $F$ peak continues to grow, and the case of $U_{b}=-10$ (black) is still higher than the other cases, but the magnitude is now only 5-6 times higher, instead of almost 10 times in the case of Figure 1(b). The harmonic emission also continues to grow in amplitude, and the trend seen in Figure 1(b) still holds, that is, the case of equal beam speeds, $U_{b}=-U_{f}=-8$ (red), seems to have the highest peak, but the other cases also show a continued increase in the peak of the $H$ mode, so that the differences are not so significant as in Figure 1(b). It is seen that the $H$ peak generated in the case $U_{b}=-10$ (black) approaches that generated in the case $U_{b}=-8$.

Figure 1(d) depicts the time step at $\tau=2000$. It is seen that the $F$ peak continues to increase when compared to $\tau=1000$, but not by very much. The case of $U_{b}=-10$ (black) still maintains the highest magnitude among the three cases. The $F$ mode peaks for $U_{b}=-6$ and $U_{b}=-8$ are quite comparable, however, with a slight dominance of the peak obtained with $U_{b}=-8$. The $H$ mode peaks feature magnitudes that are comparable with those of the $F$ mode peaks. Now the $H$ mode peak is most pronounced in the case of $U_{b}=-10$ (black) so that it exceeds the $H$ mode peak for $U_{b}=-8$. This shows that while the symmetric beam promoted faster excitation of $H$ emission, the asymmetric case with higher backward beam speed eventually catches up and exceeds the symmetric case, since the latter has more free energy. It is also interesting to note that by the time the system has evolved to $\tau=2000$ even the third harmonic has begun to grow, with a magnitude that is higher for the higher backward beam speed.

In Figure 2, we investigate the effect of variation on the relative density associated with the counter-propagating beams. We consider equal counter-propagating beam speeds, $U_{f}=-U_{b}=10$, and vary relative densities, using four different combinations of the relative beam densities: (i) $N_{f}=10^{-3} \quad$ and $\quad N_{b}=0, \quad$ (ii) $\quad N_{f}=8 \times 10^{-4} \quad$ and $N_{b}=2 \times 10^{-4}$, (iii) $N_{f}=6 \times 10^{-4}$ and $N_{b}=4 \times 10^{-4}$, (iv) $N_{f}=N_{b}=5 \times 10^{-4}$.

The first case corresponds to a single forward beam, while the last case depicts symmetric beams with equal beam densities and equal magnitudes for the beam speed. Figure 2(a) shows the time evolution at $\tau=100$. Again, note the broadband background radiation, which is already well established by this time, even though the initial level of $T$ mode is zero. The peak corresponding to fundamental emission is also evident. The peak is highest for the case of a single beam and decreases progressively as the backward beam density increases and the forward beam density decreases.

For $\tau=500$, the results are shown in Figure 2(b). At this time the harmonic peak is quite considerable, but the case of equal densities (black curve) exhibits the fastest growth, which 
is opposite to the behavior observed for the fundamental emission. The cases of $60 \%$ forward (and $40 \%$ backward) beam and equal densities show very similar results (black and green curves) such that the two curves almost overlap. For increasing difference between the beam densities, the $H$ peak continues to decrease, so that in the case of a single beam the magnitude is almost an order of magnitude less than that attained in the case of beams of equal (or nearly equal) density.

Figure 2(c) shows the situation at $\tau=1000$, where it is seen that both $F$ and $H$ emission peaks continue to develop, reaching comparable levels. The magnitude of the $H$ peak is still lower in the case of a single beam, but the magnitude is closer to that of opposite beams with similar densities, which becomes apparent when it is compared with Figure 2(b) for $\tau=500$.

Figure 2(d) corresponds to $\tau=2000$, where the third harmonic peak becomes visible and is dominant in the case of a single, denser, beam. It is seen that at $\tau=2000$ the $H$ peak is higher in the case of a single beam, in contrast to what has been observed at earlier times. It seems that the occurrence of opposite beams of comparable densities hastens the growth of the $H$ peak, compared to the case of a single beam, but the final intensity attained at the harmonic tends to be larger for a more intense single beam.

The total wave energy density for each mode as a function of $\tau$ can be obtained by integrating the normalized intensity $\mathcal{E}_{a}^{\alpha}(\tau)$ over pitch angle $\theta$ and wavenumber $q$,

$$
\mathcal{E}_{\alpha}(\tau)=\int d \theta \int d q q \mathcal{E}_{q}^{\alpha}(\tau)
$$

In Figure 3 we show the time evolution of the total wave energy densities for each mode. Shown in the left-hand panels, from top to bottom, are the time evolution of $L, S$, and $T$ waves, for which we fixed $N_{f}=6.0 \times 10^{-4}, N_{b}=4.0 \times 10^{-4}$, $U_{f}=8.0$, and varied the backward beam speed, $U_{b}=-6.0$, -8.0 , and -10.0 , as in Figure 1. The right-hand panels are the same in that, going from top to bottom, we plot energy densities of $L, S$, and $T$ waves as a function of time, except that for these panels we fix the beam velocities as $U_{f}=10.0$, $U_{b}=-10.0$, and vary the relative beam densities: (i) $\left(N_{f}, N_{b}\right)$ $=\left(0.5 \times 10^{-4}, 0.5 \times 10^{-4}\right), \quad$ (ii) $\left(N_{f}, N_{b}\right)=\left(0.6 \times 10^{-4}\right.$, $\left.0.4 \times 10^{-4}\right)$, (iii) $\left(N_{f}, N_{b}\right)=\left(0.8 \times 10^{-4}, 0.2 \times 10^{-4}\right)$, (iv) $\left(N_{f}, N_{b}\right)=\left(1.0 \times 10^{-4}, 0.0 \times 10^{-4}\right)$. These cases are the same as in Figure 2.

The top two panels of Figure 3 show the time evolution of the energy density of $L$ mode waves, which displays an exponential increase and quasi-saturation around $\tau \approx 300$ or so. It is seen that the case of higher backward beam speed $U_{b}=-10.0$ produces the highest saturated $L$ mode wave energy, which is expected, but the variation of relative beam densities does not show any appreciable change in the saturated $L$ mode wave energy. Instead, the top-right panel shows that a single beam leads to the fastest exponential growth of the $L$ mode and that the symmetric counter-streaming beams lead to the slowest onset of instability. For the energy densities of $S$ mode waves, the middle panels show that the relative growth is much smaller than that of the $L$ mode. The middle panel on the left shows that the increase in the energy in the $S$ waves is more pronounced if the speed of the backward beam is equal to that of the forward beam, with the smaller growth occurring for the slower backward beam $\left(U_{b}=-6.0\right.$, in the case depicted in Figure 3 ). The middle panel on the right of Figure 3 shows that the growth of $S$ wave energy tends to be faster in the case of a single beam than in the case of opposite beams where the total number density of beam particles is shared.

The bottom panels of Figure 3 show the time evolution of $T$ waves. It is seen that the $T$ mode grows from zero initial intensity to a finite value almost immediately. This is the onset of the spontaneous emission of background radiation as predicted by the nonlinear wave-particle scattering term (TsTL) (Ziebell et al. 2014, 2015). The overall trend in the time evolution of radiation energy is in agreement with the time evolution of longitudinal modes, $L$ and $S$. For the highest backward beam velocity, $U_{b}=-10.0$, more particle free energy is available, and so the radiation is most intense. In the case of fixed beam speeds, changing the relative beam densities again does not lead to any perceivable change in the radiation energy, but the onset time is slightly earlier for the case of a single beam.

On the basis of the findings thus far one may infer that the overall dynamics of the beam-plasma system does not depend too critically on whether there exists a single beam or whether there is a secondary beam, which is related to more subtle differences in the time evolution of the system. As we shall see next, these more subtle effects of the presence of counterstreaming beams can be seen most clearly if we analyze the time evolution of the radiation intensity at each harmonic.

Thus in Figure 4 we show the time evolution of radiation energy at each harmonic of the $T$ wave spectrum. The panel layout is the same as in Figure 3, namely, the left-hand panels show the dependence on the backward beam speed, while the right-hand panels display variations with relative beam densities. However, unlike Figure 3, the top two panels now depict the "fundamental" $(F)$ emission, with wave frequency close to $\omega_{p e}$, while the middle and bottom panels depict the second and third harmonic modes, with frequencies close to $2 \omega_{p e}$ and $3 \omega_{p e}$, respectively.

First, it is apparent from the top panels that as far as $F$ emission is concerned, for fixed beam densities, the backward beam with highest speed leads to the highest radiation level, and for fixed beam energy, the single beam leads to the fastest generation of radiation. Regarding the $H$ emission, in the leftmiddle panel one can clearly see that the case of $U_{b}=-8.0$ has the fastest growth rate when compared with the other two cases (although the case of strongest backward beam $U_{b}=-10.0$ eventually exceeds the case of $U_{b}=-8.0$ in radiation intensity over a long time). Since the forward beam has $U_{f}=8.0$, the backward beam with $U_{b}=-8.0$ (red curve) represents the situation with identical counter-streaming beams. The rightmiddle panel, which shows the dependence on the relative beam densities, shows that the fastest growth occurs with two opposite beams of similar characteristics, but that the asymptotic state is such that the higher $H$ emission occurs for the case of a single, more intense, beam. In fact, the case of equal beam densities, shown in blue, corresponds to the fastest $H$ emission in time. The third harmonic emission, appearing in the bottom panels of Figure 4, on the other hand, displays almost no dependence on the variation in backward beam speed and a small change due to the relative beam densities.

\section{SUMMARY AND CONCLUSIONS}

It is commonly accepted that the fundamental radiation emission mechanism responsible for both type-II and type-III solar and interplanetary radio bursts is plasma emission. While 

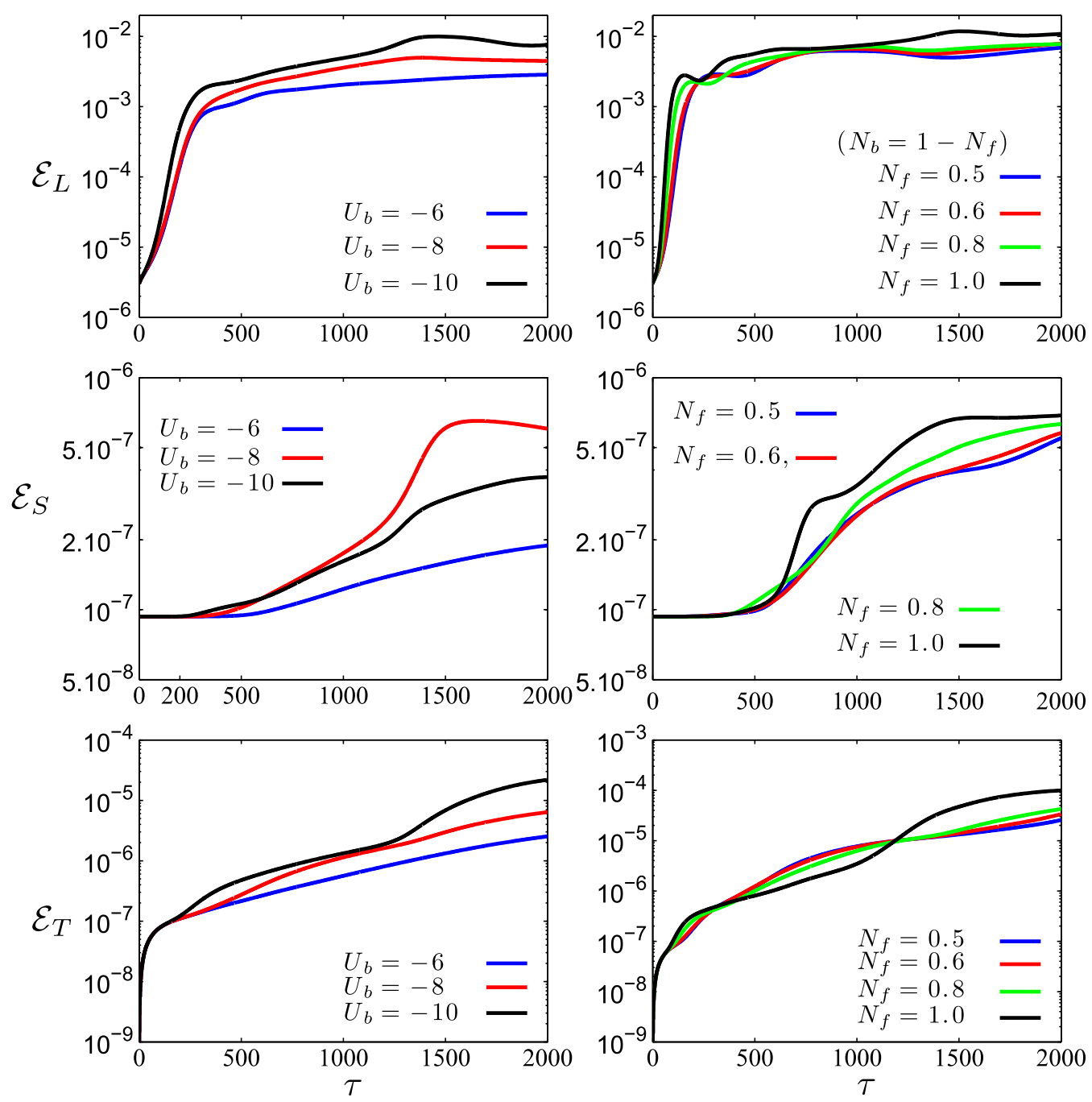

Figure 3. Integrated wave intensity, $\mathcal{E}_{\alpha}=\int d \theta \int d q q \mathcal{E}_{q}^{\alpha}$, as a function of normalized time, $\tau$. Top left- and right-hand panels are for Langmuir waves $(\alpha=L)$; the middle two panels plot ion-sound wave intensity $(\alpha=S)$; and the bottom panels correspond to transverse waves $(\alpha=T)$. The left-hand column shows the case of fixed beam densities, $\left(N_{f}, N_{b}\right)=\left(6.0 \times 10^{-4}, 4.0 \times 10^{-4}\right)$ and fixed forward beam speed, $U_{f}=8.0$, while the backward beam speed is varying: $U_{b}=-6.0$ (blue), $U_{b}=-8.0$ (red), and $U_{b}=-10.0$ (black). The right-hand column shows the variation in relative number densities: $\left(N_{f}, N_{b}\right)=\left(0.5 \times 10^{-4}, 0.5 \times 10^{-4}\right)$ (blue), $\left(N_{f}, N_{b}\right)=\left(0.6 \times 10^{-4}, 0.4 \times 10^{-4}\right)($ red $),\left(N_{f}, N_{b}\right)=\left(0.8 \times 10^{-4}, 0.2 \times 10^{-4}\right)($ green $),\left(N_{f}, N_{b}\right)=\left(1.0 \times 10^{-4}, 0.0 \times 10^{-4}\right)($ black $)$, for $U_{f}=10.0, U_{b}=-10.0$.

type-III bursts are associated with the flare-generated energetic electrons streaming out of the source region along open magnetic field lines, it is generally thought that type-II sources are related to the CME shock and the accelerated electrons in the foreshock region. Recently, Ganse et al. (2012a, 2012b) noted that the CME shock front spans a large spatial area that encompasses varying local plasma frequency. This implies that the type-II bursts should have a broad frequency band, which is in contrast to observed narrow-band type-II emissions. They proposed a model where actual type-II sources might be confined to a special CME foreshock region that has a double hump structure. The counter-streaming electrons that may exist between the two structures may preferentially excite radiation because the nonlinear coalescence processes are facilitated by the presence of two beams. Ganse et al. (2012a, 2012b) carried out particle-in-cell (PIC) simulations to demonstrate this point. Note that Umeda (2010) also compared the property of $H$ emission for single versus counter-streaming beams, but his work stands alone as a fundamental study of plasma emission, rather than within the context of type-II radio bursts.
Nevertheless, Umeda's finding is consistent with Ganse et al. (2012a, 2012b).

In the present paper we addressed the same problem based upon EM weak turbulence theory. We found that in an overall sense, neither the plasma emission nor the beam-plasma interaction process that involves Langmuir and ion-sound turbulent dynamics shows any great dependence on the presence of the secondary beam. However, if we break down the radiation emission into individual harmonics, then the numerical solution reveals the subtle dependence on the details of the counter-streaming beams. To be specific, we found that the fundamental emission increases in intensity if the density and the forward beam speed are fixed and simply the backward beam speed is increased. Similarly, if the beam speeds are fixed, the fundamental emission is most intense when there is only one beam. The second harmonic emission, on the other hand, shows that the fastest development occurs when the counter-streaming beams are identical in density and speed, but not in the direction of the beam, although the final level of emission shows that a single beam with larger density can overcome the emission due to two opposite and less dense 

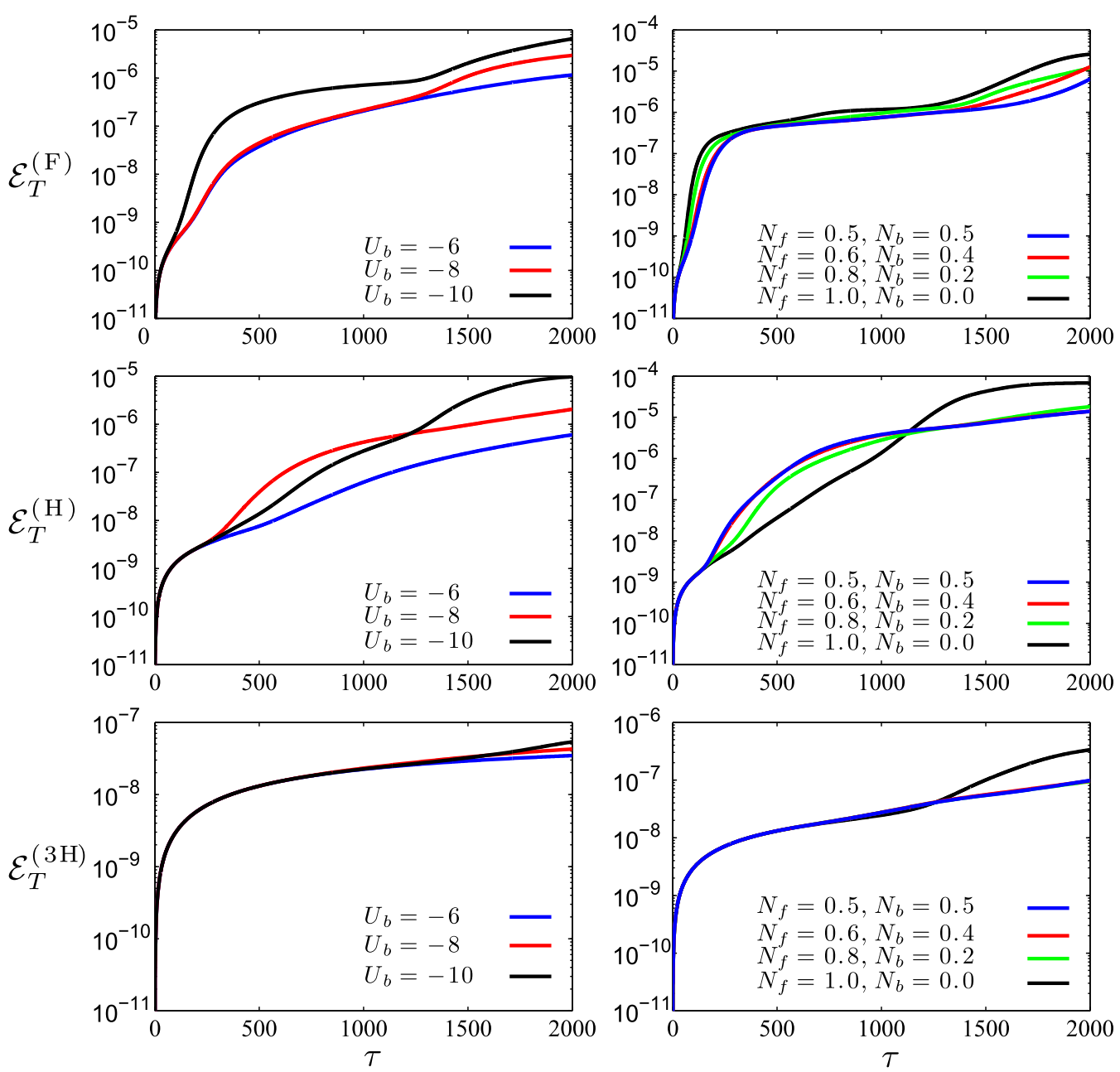

Figure 4. Integrated radiation intensity, $\mathcal{E}_{T}=\int d \theta \int d q q \mathcal{E}_{q}^{T}$, at each harmonic, as a function of normalized time, $\tau$. The top two panels are for fundamental emission $(F)$; the middle two panels represent (second) harmonic $(H)$ emission; and the bottom panels correspond to third harmonic emission (H3). The left-hand column shows the case of fixed beam densities, $\left(N_{f}, N_{b}\right)=\left(6.0 \times 10^{-4}, 4.0 \times 10^{-4}\right)$ and fixed forward beam speed, $U_{f}=8.0$, while the backward beam speed is varying: $U_{b}=-6.0$ (blue), $U_{b}=-8.0$ (red), and $U_{b}=-10.0$ (black). The right-hand column shows the variation with relative number densities: $\left(N_{f}, N_{b}\right)$ $=\left(0.5 \times 10^{-4}, 0.5 \times 10^{-4}\right)$ (blue) $,\left(N_{f}, N_{b}\right)=\left(0.6 \times 10^{-4}, 0.4 \times 10^{-4}\right)($ red $),\left(N_{f}, N_{b}\right)=\left(0.8 \times 10^{-4}, 0.2 \times 10^{-4}\right)($ green $),\left(N_{f}, N_{b}\right)=\left(1.0 \times 10^{-4}, 0.0 \times 10^{-4}\right)$ (black), for $U_{f}=10.0, U_{b}=-10.0$.

beams. The weak third harmonic emission, on the other hand, shows no clear dependence on either the variation in the backward beam speed or the variations in relative beam density.

These findings are somewhat consistent with earlier findings by Ganse et al. (2012a, 2012b) and also Umeda (2010), who found an overall increase in wave emission for the counterstreaming situation, but did not clearly distinguish the difference between the fundamental and harmonic modes.

The implication of the present work is that while our work largely confirmed and conforms with the suggestion by Ganse et al. (2012a, 2012b), the effects of counter-streaming beams on the general plasma emission are not as pronounced as envisioned by Ganse et al. (2012a, 2012b). This means the problem of why the type-II bursts are observed in relatively narrow bands while the CME shock encompasses a large spatial area with greatly varying local density remains largely unresolved.

It is worthwhile to comment on some questions related to the velocity distribution functions. One of the results of many years of in situ observations in the space environment is the knowledge that the velocity distributions of ions and electrons are frequently non-Maxwellian, featuring power-law tails that can be described by isotropic or anisotropic kappa distributions. Some theoretical results have been obtained, which show that the power-law distributions may be the outcome of nonlinear processes that are described by weak turbulence theory (Yoon 2011, 2012). For the present investigation, our interest has been the understanding of the effect of the presence of opposite beams on the evolution of the beam-plasma system in more than one dimension, and we decided to start from the conventional equilibrium state described by Maxwellian distributions. However, different configurations are under investigation, including cases where the equilibrium is already characterized by a kappa-like distribution, prior to the injection of the beam. We intend to report on the results of these investigations in the near future.

This work has been partially supported by the Brazilian agencies $\mathrm{CNPq}$ and FAPERGS. P.H.Y. acknowledges the BK21 plus grant from the National Research Foundation (NRF) of the Republic of Korea to Kyung Hee University. P.H. Y. also gratefully acknowledge the GFT Foundation Science Award. Part of this work was carried out while P.H.Y. was 
visiting Ruhr University Bochum, Germany, which was made possible by support from the Ruhr Universität Bochum Research School PLUS, funded by Germany's Excellence Initiative (DFG GSC 98/3).

\section{REFERENCES}

Cairns, I. H. 1987a, JPIPh, 38, 169

Cairns, I. H. 1987b, JPIPh, 38, 179

Cairns, I. H. 1987c, JPIPh, 38, 199

Cane, H. V., Erickson, W. C., \& Prestage, N. P. 2002, JGR, 107, 1315

Cane, H. V., Sheeley, J., \& Howard, R. A. 1987, JGR, 92, 9869

Dulk, G. A. 1985, ARA\&A, 23, 169

Fainberg, J., \& Stone, R. G. 1974, SSRv, 16, 145

Ganse, U., Kilian, P., Spanier, F., \& Vainio, R. 2012a, ApJ, 751, 145

Ganse, U., Kilian, P., Vainio, R., \& Spanier, F. 2012b, SoPh, 280, 551

Ginzburg, C. L., \& Zheleznyakov, V. V. 1958, SvA, 2, 653

Goldman, M. V. 1983, SoPh, 89, 403

Goldman, M. V., \& Dubois, D. F. 1982, PhFl, 25, 1062

Kaplan, S. A., \& Tsytovich, V. N. 1968, SvA, 11, 956

Karlický, M., \& Vandas, M. 2007, P\&SS, 55, 2336

Kasaba, Y., Matsumoto, H., \& Omura, Y. 2001, JGR, 106, 18693

Kundu, M. R. 1965, Solar Radio Astronomy (New York: Interscience)

Li, B., Cairns, I. H., \& Robinson, P. A. 2008a, JGR, 113, A06104

Li, B., Cairns, I. H., \& Robinson, P. A. 2008b, JGR, 113, A06105

Li, B., Cairns, I. H., \& Robinson, P. A. 2009, JGR, 114, A02104

Li, B., Cairns, I. H., \& Robinson, P. A. 2010, A\&A, 510, L6

Li, B., Cairns, I. H., \& Robinson, P. A. 2011a, ApJ, 730, 20

Li, B., Cairns, I. H., \& Robinson, P. A. 2011b, ApJ, 730, 21

Li, B., Cairns, I. H., Yan, Y. H., \& Robinson, P. A. 2011c, ApJL, 738, L9

Li, B., Robinson, P. A., \& Cairns, I. H. 2006a, PhRvL, 96, 145005

Li, B., Robinson, P. A., \& Cairns, I. H. 2006b, PhPl, 13, 092902

Li, B., Willes, A. J., Robinson, P. A., \& Cairns, I. H. 2005a, PhP1, 12, 052324

Li, B., Willes, A. J., Robinson, P. A., \& Cairns, I. H. 2005b, PhPl, 12, 012103

Lin, R. P. 1974, SSRv, 16, 189

Melrose, D. B. 1980a, Plasma Astrophysics, Vol. 1, 2 (New York: Gordon and Breach)
Melrose, D. B. 1980b, SSRv, 26, 3

Melrose, D. B. 1982, AuJPh, 35, 67

Melrose, D. B. 1986, Instabilities in Space and Laboratory Plasmas (New York: Cambridge Univ. Press)

Melrose, D. B. 1987, SoPh, 111, 89

Ratcliffe, H., Bian, N. H., \& Kontar, E. P. 2012, ApJ, 761, 176

Reid, H. A. S., \& Ratcliffe, H. 2014, RAA, 14, 773

Reiner, M. J., Goetz, K., Fainberg, J., et al. 2009, SoPh, 259, 255

Reiner, M. J., Stone, R. G., \& Fainberg, J. 1992, ApJ, 394, 340

Rhee, T., Ryu, C.-M., Woo, M., et al. 2009a, ApJ, 694, 618

Rhee, T., Woo, M., \& Ryu, C.-M. 2009b, JKPS, 54, 313

Roberts, J. A. 1959, AuJPh, 12, 327

Robinson, P. A., \& Cairns, I. H. 1998a, SoPh, 181, 363

Robinson, P. A., \& Cairns, I. H. 1998b, SoPh, 181, 395

Robinson, P. A., \& Cairns, I. H. 1998c, SoPh, 181, 429

Rosenberg, H. 1976, RSPTA, 281, 461

Smerd, S. F. 1976, SoPh, 46, 493

Stewart, R. T. 1974, in Proc. IAU Symp. 57, Coronal Disturbances, ed. G. Newkirk (Dordrecht: Reidel), 161

Suzuki, S., \& Dulk, G. A. 1985, in Solar Radiophysics, ed. D. J. McLean, \& N. R. Labrum (New York: Cambridge Univ. Press), 289

Tsytovich, V. N. 1967, SvPhU, 9, 805

Umeda, T. 2010, JGR, 115, A01204

Wild, J. P. 1950a, AuSRA, 3, 387

Wild, J. P. 1950b, AuSRA, 3, 541

Wild, J. P., \& McCready, L. L. 1950, AuSRA, 3, 399

Wild, J. P., Murray, J. D., \& Rowe, W. C. 1954, AuJPh, 7, 439

Wild, J. P., Sheridan, K. V., \& Neylan, A. A. 1959, AuJPh, 12, 369

Wild, J. P., \& Smerd, S. F. 1972, ARA\&A, 10, 159

Wild, J. P., Smerd, S. F., \& Weiss, A. A. 1963, ARA\&A, 1, 291

Yoon, P. H. 2011, PhPl, 18, 122303

Yoon, P. H. 2012, PhPl, 19, 012304

Zheleznyakov, V. V. 1970, Radio Emission of the Sun and Planets (New York: Pergamon)

Zheleznyakov, V. V., \& Zaitsev, V. V. 1970, SvA, 14, 250

Ziebell, L. F., Yoon, P. H., Gaelzer, R., \& Pavan, J. 2014, ApJL, 795, L32

Ziebell, L. F., Yoon, P. H., Petruzzelis, L. T., Gaelzer, R., \& Pavan, J. 2015, ApJ, 806, 237 